\title{
A mixed precision semi-Lagrangian algorithm and its performance on accelerators
}

\author{
Lukas Einkemmer
}

\begin{abstract}
In this paper we propose a mixed precision algorithm in the context of the semi-Lagrangian discontinuous Galerkin method. The performance of this approach is evaluated on a traditional dual socket workstation as well as on a Xeon Phi and a NVIDIA K80. We find that the mixed precision algorithm can be implemented efficiently on these architectures. This implies that, in addition to the considerable reduction in memory, a substantial increase in performance can be observed as well. Moreover, we discuss the relative performance of our implementations.
\end{abstract}

Index Terms-Mixed precision, Semi-Lagrangian methods, Accelerators, GPU, Intel Xeon Phi

\section{INTRODUCTION}

Semi-Lagrangian methods are an important class of numerical algorithms to solve hyperbolic partial differential equations. These methods do not suffer from a Courant-FriedrichsLewy (CFL) condition and can be applied without solving a linear system of equations. Consequently, semi-Lagrangian methods are computationally attractive and have been applied to a wide variety of problems ranging from weather forecasting to plasma simulations.

Due to the prevalence of these algorithms in applications, studying semi-Lagrangian methods and their efficient implementation is an important research area. In particular, in plasma physics applications problems in a high dimensional setting (up to 6 dimensions; three position and three velocity directions) are common. Thus, both memory consumption and computational performance are a major concern in such simulations.

Let us note that contrary to the much studied stencil codes, these algorithms, in general, do not have pre-determined memory access patterns and in some cases have non-uniform degrees of freedom (i.e. the specific computation performed depends on index of the data under consideration). This potentially presents a problem for accelerators as aligned data access is usually required in order to obtain optimal performance.

The crucial part in any semi-Lagrangian algorithm is the interpolation or projection step. In this step the translated function is interpolated/projected back to the grid/approximation space. In the literature a number of numerical schemes have been introduced. Most commonly, cubic spline interpolation is employed in practice. However, recently the so-called semiLagrangian discontinuous Galerkin scheme has been considered. This method is competitive with spline interpolation but is a completely local numerical method. This fact greatly facilitates the implementation on parallel architectures (both on shared as well as on distributed memory systems).

L. Einkemmer is with the University of Innsbruck, Austria

E-mail: lukas.einkemmer@uibk.ac.at
Due to the constraints outlined above and since the algorithm is memory bound, implementing it using single precision floating point arithmetic would significantly improve performance as well as reduce memory consumption. This is particularly true on accelerators, such as graphic processing units (GPUs) and the Intel Xeon Phi, for which memory is a more scarce resource compared to traditional central processing unit (CPU) based systems. In addition, since the memory available per core will most likely continue to decrease on future architectures, the problem of memory scarcity is going to become an even bigger issue in the future.

However, since in many practical simulations a large number of time steps, and consequently a large number of projections, have to be performed, conservation of mass at least up to double precision accuracy has been the gold standard in the physics community. This is particularly important as for the classic time-splitting approach conservation of mass implies conservation of momentum as well as a range of other desirable properties.

Mixed precision algorithms have attracted some interest recently. This is particularly true for numerical linear algebra algorithms (see, for example, [1], [10], [18], [12], [13], [3]). For example, in the context of iterative methods it is very natural to compute a first approximation in single precision which is then refined, if necessary, using double precision arithmetics. Also computing the preconditioner in single precision to increase performance is an often used technique. Furthermore, mixed precision algorithms for some specific applications have been proposed as well (see, for example, [12], [17]) and even the possibility to automatically convert certain parts of computer programs to single precision has been investigated (see [16]). However, the traditional formulation of semi-Lagrangian methods poses significant difficulties if a mixed precision implementation is to be considered.

In this paper we propose a mixed precision semi-Lagrangian approach that succeeds in conserving the mass up to double precision while storing almost all of the data used in the computation as single precision floating point numbers. We use the before mentioned semi-Lagrangian discontinuous Galerkin method, but instead of storing certain function evaluations at a non-equidistant grid (as has been the predominant approach in the literature), we use the fact that the corresponding approximation can be written as an expansion in the Legendre polynomials. The first coefficient in this expansion is simply the mass in a subset of the computational domain (which is then stored in double precision). Furthermore, we will investigate the performance of this algorithm on an Intel Xeon Phi and a NVIDIA K80 GPU and compare the performance attained to a dual socket workstation. 
The remainder of this paper is structured as follows. In section ПI we discuss the problem as well as the algorithm proposed to solve it in more detail. In addition, we briefly discuss the hardware architectures used in our implementation. In section [III we study the error of the proposed algorithm, the different implementations, and our findings with respect to performance. These can be summarized as follows.

- The semi-Lagrangian discontinuous Galerkin scheme can be implemented efficiently using mixed precision arithmetics, resulting in both a significant memory reduction as well as a significant performance increase. This is true across all the hardware architectures considered here (dual socket workstation, Intel Xeon Phi, and NVIDIA K80 GPU).

- For traditional CPU based systems our implementation, in most of the cases considered, is close to the theoretical attainable performance for memory bound problems on that architecture.

- For the Xeon Phi we observe a speed up of approximately $50 \%$ compared to the CPU implementation (using the code that has been optimized for the CPU system). Thus, in this case the Xeon Phi can act as a drop-in replacement.

- For the K80 we observe a speedup of approximately 2.5 compared to the CPU implementation. This corresponds to $75 \%$ of the peak performance for that architecture.

Finally, we conclude in section IV]

\section{BACKGROUND \& MOTIVATION}

The Vlasov equation (here stated in $1+1$ dimensions)

$$
\partial_{t} f(t, x, v)+v \partial_{x} f(t, x, v)+E(x) \partial_{v} f(t, x, v)=0
$$

models the time evolution of a plasma system. The sought after quantity is the particle density function $f(t, x, v)$, and the electric field is denoted by $E(x)$. This model is important for the description of non thermalized plasmas; i.e. plasmas where fluid models (such as magnetohydrodynamics) are not applicable. In the seminal paper by Cheng \& Knorr [4] it was recognized that by applying a time splitting approach, the Vlasov equation is reduced to a sequence of one-dimensional advections. That is, in order to solve the Vlasov equation efficiently it is necessary to develop a good integrator for

$$
\partial_{t} u(t, x)+a \partial_{x} u(t, x)=0,
$$

where $a \in \mathbb{R}$ is a constant. Let us emphasize that the simplicity of this equation (for which even an analytic solution can be derived) is deceiving. In fact, a large body of literature has been devoted to this problem (see, for example, [22], [11], [5], [20], [19]). For solving the Vlasov equation it is important that the numerical scheme is free of a CFL condition. Otherwise, the numerical scheme would be forced to take excessively small time steps. Therefore, so-called semiLagrangian methods have become popular in this field. These methods follow the characteristics backward in time in order to compute the function values at the grid points. Note, however, that the feet of the characteristics do not necessarily coincide with the grid. Thus, an interpolation procedure has to be employed. Note that in the case where the characteristics can be determined analytically (as is the case for equation (1)) this is the only approximation made (except for the time splitting).

Consequently it is important to choose a suitable interpolation scheme. An obvious choice is to use local polynomial interpolation or methods based on fast Fourier techniques. Especially the latter was used quite extensively in many Vlasov simulations (see, for example, [4], [15]). However, more recently interpolation using cubic splines has become the de facto standard [6] and the performance of numerical software packages that implement such methods has been investigated in some detail (see, for example, [21], [2], [14]).

This is due to the fact that spline interpolation is mass conservative and shows little numerical diffusion compared to alternative approaches. In addition, it is not as prone to oscillations (and thus to the appearance of negative values) as are Fourier techniques.

However, the procedure also suffers from a number of shortcomings. Most notably that it is a global algorithm. That is, the construction of the cubic spline (for which we have to solve a sparse linear system) couples each degree of freedom with each other degree of freedom. The resulting all-to-all communication is a serious issue with respect to the scalability of such algorithms.

\section{A. Description of the algorithm}

In recent years an alternative method has emerged. The socalled semi-Lagrangian discontinuous Galerkin scheme was independently proposed by [5], [20], [19]. Since a variant of this method will be used in the present paper, we will describe it in some detail. First, the computational domain is divided into a number of cells $C_{i}=\left[x_{i-1 / 2}, x_{i+1 / 2}\right]$. In each of these cells we approximate the exact solution by a polynomial of degree $p$. This approximation requires the storage of $o=p+1$ degrees of freedom. Let us note that no continuity constraint is enforced at the cell boundaries. Thus, the approximation of the continuous solution $u(t, x)$ is performed by a function with discontinuities at the cell interfaces. The corresponding jumps are bounded in magnitude by the discretization error. To solve equation (1) we translate the approximant (this can be done analytically) and then perform an $L^{2}$ projection to the subspace of piecewise polynomials up to degree $p$. This can be easily accomplished by choosing an orthogonal basis of the polynomial approximation space and results in an approximation of order $o$.

The semi-Lagrangian discontinuous Galerkin method is mass conservative by construction and compares very favorably to the cubic spline interpolation (see, for example, [5], [8], [23], [9]). In addition, it requires at most the data from two adjacent cells in order to compute the approximation at the subsequent time step (note that this behavior is independent of the CFL number). Thus, the semi-Lagrangian discontinuous Galerkin method is a completely local scheme. This greatly facilitates the implementation on distributed memory systems (see, for example, [7]).

In most of the literature, the Lagrange basis polynomials at the Gauss-Legendre quadrature nodes are used as the basis. This has the advantage that the degrees of freedom are function 
evaluations on a non-equidistant grid. However, since the degrees of freedom correspond to function evaluations, even for smooth functions, all of them are approximately equal in magnitude. Thus, this representation is unsuitable in the context of a mixed precision implementation.

In order to remedy this issue we use the fact that the first $o$ Legendre polynomials (appropriately scaled to the corresponding cell size) form a basis of the space of polynomials up to degree $p$. Thus, in order to approximate $u(x)$ in the cell $[-h / 2, h / 2]$ we use

$$
u(x) \approx \sum_{j=0}^{p} c_{j} P_{j}\left(\frac{2}{h} x\right),
$$

where $P_{j}$ is the $j$ th Legendre polynomial defined on the interval $[-1,1]$. The degrees of freedom, which are consequently stored in computer memory, are the coefficients $c_{j}$ that appear in the expansion. These coefficients can be computed as follows

$$
c_{j}=\frac{2 j+1}{2} \frac{2}{h} \int_{-h / 2}^{h / 2} u(x) P_{j}\left(\frac{2}{h} x\right) \mathrm{d} x .
$$

This integral is easily solved exactly by performing a GaussLegendre quadrature. The advantage of this representation is that for a smooth function $u$ we have $c_{j} \sim h^{j}$. Thus, $c_{0}$ will be $\mathcal{O}(1)$ while higher order coefficients become progressively smaller. This scaling can be exploited by storing the higher order coefficients with less precision (i.e. using single precision). This can be interpreted as a, in general lossy, compression scheme where less important coefficients are stored using fewer bytes.

Let us also note that $c_{0}$ corresponds to the mass in $[-h / 2, h / 2]$. This is especially convenient for a mixed precision implementation as storing $c_{0}$ in double precision (and the remaining coefficients in single precision) automatically ensures conservation of mass up to double precision accuracy.

In the present text we will not derive the implemented algorithm in any detail. What is important for the present discussion, however, is that by performing the Gauss-Legendre quadrature of equation (2) we obtain the following update rule for the coefficient $c_{i j}^{n+1}$ at time $t_{n+1}(i$ is the cell index)

$$
c_{i j}^{n+1}=\sum_{l} A_{j l} c_{i^{\star} l}^{n}+\sum_{j} B_{j l} c_{i^{\star}+1 ; l}^{n}
$$

where $A \in \mathbb{R}^{o \times o}$ and $B \in \mathbb{R}^{o \times o}$ are matrices that only depend on the time step size and $i^{\star}$ is the integer part of the CFL number. Note that the CFL number is not a fixed quantity but can depend, for example, on the electric field. Thus, it is not a-priori known by how much the index $i$ is translated in order to obtain $i^{\star}$.

The matrices can be precomputed at the beginning of each time step and thus their cost will not be a major concern except for an extremely coarse space discretization. Furthermore, the size of the matrices is negligible in comparison to the cache size and it is thus reasonable to assume that access to these matrices is quick. Consequently, the algorithm requires (assuming a perfect cache) one memory load and one memory store per degree of freedom, which we have to compare to the $4 o-1$ arithmetic instructions. Thus, even for a sixth-order method the flop/byte ratio is only about 1.4 for double precision and 2.9 for single precision. This is significantly below the flop/byte ratio for all the computer systems considered here (see Table 【).

TABLE I

HARDWARE CHARACTERISTICS OF THE DUAL SOCKET WORKSTATION USED IN THE NUMERICAL SIMULATIONS. PEAK ARITHMETIC PERFORMANCE FOR SINGLE AND DOUBLE PRECISION AND THE ATTAINABLE MEMORY BANDWIDTH AS WELL AS THE FLOP/BYTE RATIOS ARE LISTED. THE DAGGER INDICATES THAT THE BANDWIDTH ACHIEVED

\begin{tabular}{|c|c|c|c|c|c|}
\hline & \multicolumn{2}{|c|}{ TFlops/s } & \multirow[b]{2}{*}{$\mathrm{GB} / \mathrm{s}$} & \multicolumn{2}{|c|}{ flop/byte } \\
\hline & double & single & & double & single \\
\hline $2 \times$ E5-2630 v3 & 0.6 & 1.2 & 59 & 10.2 & 20.3 \\
\hline 1x Xeon Phi 7120 & 1.2 & 2.4 & $150^{\dagger}$ & 8 & 16 \\
\hline $0.5 \times \mathrm{K} 80$ & 1.5 & 4.4 & $170^{\dagger}$ & 8.8 & 25.9 \\
\hline
\end{tabular}
BY A MEMORY COPY BENCHMARK IS LISTED (AND NOT THE THEORETICAL BANDWIDTH SPECIFIED BY THE VENDOR).

However, floating point arithmetic is not the only concern. Since the algorithm requests a memory load for the addresses $i^{\star}$ and $i^{\star}+1$ in one iteration and to $i^{\star}+1$ and $i^{\star}+2$ in the next, cache performance is vital to obtain good performance. This is especially true for the CPU and Intel Xeon Phi implementation as in those instances there is little possibility to directly manipulate the cache. We will discuss a number these issues in more detail in the next section.

\section{B. Hardware architecture}

As has been mentioned before, reducing memory consumption is especially important on accelerators due to the limited amount of memory available on these systems. In the present paper we will use the Intel Xeon Phi 7120 which includes 16 GB of memory and the NVIDIA K80 which consists of two GPUs in a single package with 12 GB of memory each. The purpose of the present section is to describe these two architectures.

Let us start with the Intel Xeon Phi due its similarity with the standard $x 86$ architecture. In fact, on the Intel Xeon Phi a full Linux operating system is employed. The Xeon Phi can either be used in offload mode (where the CPU and the Xeon Phi work together and exchange data over the PCIe bus) and in standalone mode (where a program runs on the Xeon Phi without intervention from the CPU system). The preferred programming model for the Xeon Phi is OpenMP which we use in all our implementations. However, they are also important differences. The Intel Xeon Phi (similar to GPUs) uses GDDR5 memory which is optimized for applications which can exploit high memory bandwidth. However, it should be emphasized that to obtain this bandwidth certain access pattern are required. This has to be contrasted with DDR3 memory which is also fairly effective if random access patterns are used. The Xeon Phi consists of 61 cores each of which has access to an $32 \mathrm{~KB} \mathrm{L1}$ cache and an $512 \mathrm{~KB}$ L2 cache. The latter can interchange data via a ring interconnect. Let us also mention that the Xeon Phi implements eight double wide vector units (AVX512).

On the other hand, the NVIDIA K80 is a more lightweight architecture. No operating system runs on the card and thus 
computations on the GPU always have to be controlled by an application running on the host system. Similar to the Xeon $\mathrm{Phi}$, the K80 uses GDDR5 memory. Although caches are a more recent addition to GPUs, this Kepler generation card includes both an L2 cache and an L1 cache. The latter is implemented together with a so-called shared memory. Shared memory is basically an L1 cache that can be directly controlled by the program (thus it is a user managed cache). The relative size of the L1 cache and the shared memory can be configured to some extend and these two types of memory are shared by all blocks running on the same streaming multiprocessor. The K80 exposes significantly more parallelism with 2496 socalled CUDA cores. Note, however, that 32 such CUDA cores are collected in one warp and all threads in a particular warp have to execute the same instruction if optimal performance is to be achieved. Thus, one might argue that the GPU consists of 78 cores with 32 threads grouped together in a vector unit. The difference to the vector units found more commonly in CPUs, however, is the programming model. While for CPUs/Xeon Phi vectorization is mostly delegated to the compiler, the CUDA programming model exposes this behavior more directly to the programmer. An additional difference is that in principle any command can be vectorized, as long as all threads within a warp execute the same statement (i.e. there is no branch divergence). Since CUDA is the most common programming model for NVIDIA GPUs, we use it in all our implementations.

\section{RESULTS}

\section{A. Accuracy}

It is well accepted in the Vlasov community that preserving mass up to machine precision (i.e. up to double precision accuracy) is vital in order to obtain a physically reasonable solution (especially for long time integration). This is perhaps the most serious argument against using single precision floating point numbers in such simulations. However, as mentioned before, to only store the coefficient $c_{0}$ in double precision (and the remaining coefficients in single precision) is sufficient in order to retain this behavior.

The purpose of the present section is to confirm this result numerically and to investigate the different numerical schemes that result as a consequence of increasing the number of coefficients stored in double precision. To that end a number of numerical results are listed in Table II We can easily observe the expected behavior that all numerical methods which store at least $c_{0}$ in double precision conserve the mass up to that accuracy.

Let us now discuss the error in the numerical solution that is introduced by storing some coefficients in single precision. This error is computed by comparing the numerical scheme under investigation to the double precision implementation. The corresponding results are given in Table III. We observe that for the smooth initial value storing $c_{0}$ in double precision (and all other coefficients in single precision) already yields very good accuracy.

For the oscillatory initial value storing more coefficients in double precision has some effect. Note, however, that storing
TABLE II

THE ERROR (COMPARED TO THE DOUBLE PRECISION IMPLEMENTATION) AND THE ERROR IN MASS IS SHOWN FOR A SMOOTH INITIAL VALUE (TOP) AND AN OSCILLATORY INITIAL VALUE (BOTTOM). IN BOTH CASES $10^{4}$ TIME STEPS ARE CONDUCTED AND THE ERROR IS MEASURED IN THE DISCRETE $L^{2}$ NORM. THE NUMBER OF COEFFICIENTS THAT ARE STORED IN DOUBLE PRECISION IS GIVEN IN THE SECOND COLUMN.

\begin{tabular}{ccrr} 
order & \# double & error & error (mass) \\
\hline 2 & 1 & $8.98 \mathrm{e}-10$ & $4.44 \mathrm{e}-15$ \\
& 0 & $1.09 \mathrm{e}-05$ & $2.56 \mathrm{e}-06$ \\
4 & 3 & $6.16 \mathrm{e}-14$ & $1.21 \mathrm{e}-14$ \\
& 2 & $6.41 \mathrm{e}-13$ & $1.42 \mathrm{e}-14$ \\
& 1 & $3.55 \mathrm{e}-10$ & $6.15 \mathrm{e}-15$ \\
& 0 & $2.80 \mathrm{e}-05$ & $1.31 \mathrm{e}-05$ \\
order & \# double & error & error (mass) \\
\hline 2 & 1 & $9.22 \mathrm{e}-10$ & $1.04 \mathrm{e}-14$ \\
& 0 & $5.98 \mathrm{e}-06$ & $2.36 \mathrm{e}-06$ \\
4 & & & \\
& 3 & $1.22 \mathrm{e}-12$ & $1.11 \mathrm{e}-14$ \\
& 2 & $6.32 \mathrm{e}-10$ & $1.06 \mathrm{e}-14$ \\
& 1 & $6.05 \mathrm{e}-08$ & $1.64 \mathrm{e}-14$ \\
& 0 & $6.54 \mathrm{e}-05$ & $9.81 \mathrm{e}-06$
\end{tabular}

only $c_{0}$ in single precision already yields a three order of magnitude improvement as well as ensures mass conservation up to double precision accuracy. Let us also remark that the error committed by the numerical approximation (i.e. by the projections performed in course of the time integration) are large compared to the roundoff errors (except for the single precision implementation).

From a practical point of view it seems that there are few reasons to store more than one or two coefficients in double precision. Thus, in the following sections we will mostly focus on the numerical scheme were either $c_{0}$ is stored in double precision (for the second and fourth order scheme) and the scheme where $c_{0}$ and $c_{1}$ are stored in double precision (for the fourth order scheme).

To conclude this section let us note that storing only $c_{0}$ in memory results in a reduction in memory by a factor of 1.6. Note, however, that this effect becomes more pronounced in higher dimensions. For example, in two dimensions the reduction in memory is already a factor of 1.8 and in dimension three a factor of 1.97 . The latter is certainly almost indistinguishable from a pure single precision implementation, where we obtain a reduction by a factor of 2 . This should be kept in mind in the following discussion, even though for simplicity we only consider the one-dimensional case in this paper.

\section{B. CPU performance}

As described in section [II the algorithm under consideration is memory bound. Thus, we use the achieved bandwidth (measured in $\mathrm{GB} / \mathrm{s}$ ) as the metric of performance.

The numerical results for the CPU implementation are shown in Table IIII If we compare the bandwidth achieved to the theoretical bandwidth of that architecture (59 GB/s), we find that the algorithm performs very close to that limit. Thus, the implementation is almost optimal for both the second and the fourth order method. Let us note, however, that especially for the fourth order method we observe a slight decrease in 
TABLE III

THE PERFORMANCE ATTAINED BY THE CPU IMPLEMENTATION IS SHOWN FOR THE SECOND AND THE FOURTH ORDER SCHEME. THE NUMBER OF COEFFICIENTS THAT ARE STORED IN DOUBLE PRECISION IS LISTED FOR EACH CONFIGURATION. THE TABLE GIVES THE ACHIEVED SPEEDUP AND THE REDUCTION IN MEMORY COMPARED TO THE DOUBLE PRECISION IMPLEMENTATION.

\begin{tabular}{ccrrr} 
order & \# double & bandwidth & speedup & memorydown \\
\hline 2 & 2 & $55.2 \mathrm{~GB} / \mathrm{s}$ & - & - \\
2 & 1 & $51.3 \mathrm{~GB} / \mathrm{s}$ & 1.24 & 1.33 \\
2 & 0 & $51.9 \mathrm{~GB} / \mathrm{s}$ & 1.88 & 2.00 \\
& & & & - \\
4 & 4 & $52.8 \mathrm{~GB} / \mathrm{s}$ & - & 1.33 \\
4 & 2 & $48.5 \mathrm{~GB} / \mathrm{s}$ & 1.22 & 1.60 \\
4 & 1 & $42.2 \mathrm{~GB} / \mathrm{s}$ & 1.28 & 2.00 \\
4 & 0 & $43.4 \mathrm{~GB} / \mathrm{s}$ & 1.64 &
\end{tabular}

TABLE IV

THE PERFormance ATTAINED BY THE XEON PHi IMPLEMENTATION IS SHOWN FOR THE SECOND AND THE FOURTH ORDER SCHEME. THE NUMBER OF COEFFICIENTS THAT ARE STORED IN DOUBLE PRECISION IS LISTED FOR EACH CONFIGURATION. THE TABLE GIVES THE ACHIEVED SPEEDUP AND THE REDUCTION IN MEMORY COMPARED TO THE DOUBLE PRECISION IMPLEMENTATION.

\begin{tabular}{ccrrr} 
order & \# double & bandwidth & speedup & memorydown \\
\hline 2 & 2 & $76.4 \mathrm{~GB} / \mathrm{s}$ & - & - \\
2 & 1 & $67.3 \mathrm{~GB} / \mathrm{s}$ & 1.17 & 1.33 \\
2 & 0 & $76.2 \mathrm{~GB} / \mathrm{s}$ & 1.99 & 2.00 \\
& & & & \\
4 & 4 & $67.9 \mathrm{~GB} / \mathrm{s}$ & - & - \\
4 & 2 & $72.0 \mathrm{~GB} / \mathrm{s}$ & 1.41 & 1.33 \\
4 & 1 & $76.3 \mathrm{~GB} / \mathrm{s}$ & 1.80 & 1.60 \\
4 & 0 & $68.6 \mathrm{~GB} / \mathrm{s}$ & 2.02 & 2.00
\end{tabular}

performance as we increase the number of coefficients that are stored in single precision.

Let us note that in the present implementation the order of the method and the number of coefficients that are stored in single precision is available at compile time. This gives the compiler more information for optimization. If this is not done (i.e. if a generic implementation is considered) the performance is cut approximately in half.

\section{Xeon Phi performance}

The Xeon Phi implementation is identical to the implementation that was run on the dual socket workstation in the previous section. For the Xeon Phi it is even more important to avoid a generic implementation in favor of making all the relevant constants available at compile time. All the simulations conducted here are run in standalone mode.

The performance results are shown in Figure IV] The Xeon Phi manages to outperform the CPU based system by approximately 30 to $80 \%$ (depending on the configuration). This variance in speedup is mostly due to the performance characteristics of the CPU system (the performance across the different configurations is actually more consistent on the Xeon Phi). For the important configuration $o_{1}=1, o_{2}=3$ it is observed that the corresponding implementation outperform the double precision implementation by $80 \%$ with a reduction in memory by $60 \%$.

Let us note that we achieve only slightly more than onehalf of the theoretical performance possible on the Xeon Phi. To improve these results would most likely require a
TABLE V

THE PERFORMANCE ATTAINED BY THE SHARED MEMORY GPU IMPLEMENTATION IS SHOWN FOR THE SECOND AND THE FOURTH ORDER SCHEME. THE NUMBER OF COEFFICIENTS THAT ARE STORED IN DOUBLE PRECISION IS LISTED FOR EACH CONFIGURATION. THE TABLE GIVES THE ACHIEVED SPEEDUP AND THE REDUCTION IN MEMORY COMPARED TO THE DOUBLE PRECISION IMPLEMENTATION.

\begin{tabular}{ccrrr} 
order & \# double & bandwidth & speedup & memorydown \\
\hline 2 & 2 & $110.1 \mathrm{~GB} / \mathrm{s}$ & - & - \\
2 & 1 & $89.9 \mathrm{~GB} / \mathrm{s}$ & 1.09 & 1.33 \\
2 & 0 & $90.9 \mathrm{~GB} / \mathrm{s}$ & 1.65 & 2.00 \\
& & & & - \\
4 & 4 & $95.0 \mathrm{~GB} / \mathrm{s}$ & - & 1.33 \\
4 & 2 & $72.1 \mathrm{~GB} / \mathrm{s}$ & 1.01 & 1.60 \\
4 & 1 & $60.0 \mathrm{~GB} / \mathrm{s}$ & 1.01 & 2.00 \\
4 & 0 & $73.2 \mathrm{~GB} / \mathrm{s}$ & 1.54 &
\end{tabular}

more sophisticated implementation specifically tuned to the architecture of the Xeon Phi. It can be argued, however, that obtaining a substantial performance improvement with the same code that is used on the CPU is one of the major selling points of the Xeon Phi. Although this is only occasionally the case, it holds true for the implementation described here.

\section{GPU performance}

The GPU code described in this section has been implemented using the CUDA framework. In this programming paradigm most of the specifics of the GPU architecture are exposed to the programmer (although there are some major exceptions). In particular, it is the programmers responsibility to specify how much data each thread is given to process. As a starting point we have ported our CPU implementation to the GPU. That is, each thread is responsible for a single degree of freedom. This obviously requires some branching statements in case of the mixed precision implementation. However, since the performance of the algorithm is dictated by memory bandwidth, loosing a few cycles due to warp divergence is not a major concern. The achieved performance of the resulting code is slightly above $60 \mathrm{~GB} / \mathrm{s}$. In some sense this is disappointing as only one-third of the bandwidth available on the GPU is exploited. However, we should emphasize that this is still a fully generic code; i.e. the order of the method is not known at compile time and only one kernel is used. If we do the same on the CPU we achieve approximately $35 \mathrm{~GB} / \mathrm{s}$. Thus, we still observe some speedup on the GPU if naive implementations on both platforms are compared. In fact, the GPU implementation is even slightly faster than what is theoretically possible on the CPU. The situation is even more dramatic on the Xeon Phi, where the generic implementation achieves only a performance below $15 \mathrm{~GB} / \mathrm{s}$.

An investigation has shown that the performance of the described GPU implementation is mostly limited by cache misses. Fortunately, the CUDA architectures gives us direct access to shared memory which we use to cache all memory loads. The performance of the resulting implementation is shown in Figure $\mathrm{V}$.

We observe $95.6 \mathrm{~GB} / \mathrm{s}$ for the double precision implementation (for the fourth order scheme) and $109.7 \mathrm{~GB} / \mathrm{s}$ for the second order scheme. This is a distinctive improvement. However, the performance of the mixed precision and the 
TABLE VI

THE PERFORMANCE ATTAINED BY THE IMPROVED GPU IMPLEMENTATION IS SHOWN FOR THE FOURTH ORDER SCHEME. THE NUMBER OF COEFFICIENTS THAT ARE STORED IN DOUBLE PRECISION IS LISTED FOR EACH CONFIGURATION. THE TABLE GIVES THE ACHIEVED SPEEDUP AND THE REDUCTION IN MEMORY COMPARED TO THE DOUBLE PRECISION IMPLEMENTATION

\begin{tabular}{ccrrr} 
order & \# double & bandwidth & speedup & memorydown \\
\hline 4 & 4 & $137.9 \mathrm{~GB} / \mathrm{s}$ & - & - \\
4 & 1 & $130.1 \mathrm{~GB} / \mathrm{s}$ & 1.51 & 1.60 \\
4 & 0 & $142.5 \mathrm{~GB} / \mathrm{s}$ & 2.07 & 2.00
\end{tabular}

single precision implementation are significantly worse in terms of the achieved memory bandwidth. What we have to conclude is that while the mixed precision implementation saves memory, it does not improve the performance of the computation.

The reason for the disappointing performance of the mixed precision implementation is that since each thread only processes a single degree of freedom, the overhead for the necessary index calculations is relatively high. This is particularly a problem for the present algorithm as, contrary to say a finite difference stencil, it is important for the subsequent calculation at what location the currently processed degree of freedom resides within a given cell. Thus, the index calculations are more involved than is usually the case and can consequently not be completely hidden. This effect is more pronounced for the mixed precision and single precision implementation as less time is required to read and write to memory (which aggravates the problem of hiding this overhead).

The natural question then is how to improve the performance of this implementation. We have accomplished this by making each thread responsible for a single cell (not a single degree of freedom). This makes the index calculation easier and alleviates the overhead as more work is done per thread. We should note, however, that the resulting code is specific to each configuration. That is, one separate kernel has to be written for each configuration of the method (similar to the CPU and Xeon Phi implementation). As we can see from the results in Table VI, the performance is dramatically improved across the board. Moreover, independent of the implementation we observe approximately $130 \mathrm{~GB} / \mathrm{s}$ where the penalty for the mixed precision implementation is below $10 \%$. Thus, the mixed precision implementation not only decreases the memory consumption but also increases the performance by a similar factor.

To conclude this section, let us discuss what limits the performance of the present implementation. First, non aligned memory access is, to some extend, unavoidable in the present numerical scheme (as the data inside each cell is not a multiple of 128 byte). This results in a reduction of performance by approximately $10 \%$. Second, storing data in shared memory requires a block-wide synchronization barrier. This adds an additional overhead of approximately $10 \%$. Finally, the remaining $10 \%$ of performance loss are attributed to the fact that the required computations can not be perfectly interleaved with the memory accesses. In total our kernels achieve approximately $75 \%$ of the peak performance on the K80.

\section{CONCLUSION}

We have demonstrated that mixed precision semiLagrangian algorithms can be implemented in an efficient manner. On all the computer architectures considered (CPU, Xeon Phi, GPU) significant speedups can be observed in addition to the reduction in memory. Thus, we conclude that a mixed precision of the semi-Lagrangian discontinuous Galerkin method is a viable numerical scheme for simulations on both traditional CPU based systems as well as on modern accelerators.

\section{REFERENCES}

[1] M. Baboulin, A. Buttari, J. Dongarra, J. Kurzak, , J. Langou, P. Luszczek, and S. Tomov. Accelerating scientific computations with mixed precision algorithms. Comput. Phys. Commun., 180(12):2526-2533, 2009.

[2] J. Bigot, V. Grandgirard, G. Latu, Ch. Passeron, F. Rozar, and O. Thomine. Scaling GYSELA code beyond 32K-cores on Blue Gene/Q. In ESAIM: Proceedings, volume 43, pages 117-135. EDP Sciences, 2013.

[3] A. Buttari, J. Dongarra, J. Langou, J. Langou, P. Luszczek, and J. Kurzak. Mixed precision iterative refinement techniques for the solution of dense linear systems. Int. J. High Perform. C., 21(4):457466, 2007.

[4] C. Cheng and G. Knorr. The integration of the Vlasov equation in configuration space. J. Comput. Phys., 22(3):330-351, 1976.

[5] N. Crouseilles, M. Mehrenberger, and F. Vecil. Discontinuous Galerkin semi-Lagrangian method for Vlasov-Poisson. In ESAIM: Proceedings, volume 32, pages 211-230. EDP Sciences, 2011.

[6] E. Sonnendrücker. Numerical methods for the Vlasov equation. http://icerm.brown.edu/html/programs/sp_f11/schedules/slides_workshop_1_tutorial/Lec 2011.

[7] L. Einkemmer. High performance computing aspects of a dimension independent semi-Lagrangian discontinuous Galerkin code. To appear in Commun. Comput. Phys., 2015.

[8] L. Einkemmer. On the geometric properties of the semi-Lagrangian discontinuous Galerkin scheme for the Vlasov-Poisson equation. arXiv preprint, arXiv:1601.02280, 2016.

[9] L. Einkemmer and A. Ostermann. On the error propagation of semiLagrange and Fourier methods for advection problems. Comput. Math. Appl., 69(3):170-179, 2015.

[10] M. Emans and A. van der Meer. Mixed-precision AMG as linear equation solver for definite systems. Procedia Computer Science, 1(1):175-183, 2010.

[11] F. Filbet and E. Sonnendrücker. Comparison of Eulerian Vlasov solvers. Commun. Comput. Phys., 150(3):247-266, 2003.

[12] S.L. Glimberg, A.P. Engsig-Karup, and M.G. Madsen. A fast GPUaccelerated mixed-precision strategy for fully nonlinear water wave computations. In Proceedings of ENUMATH 2011, the 9th European Conference on Numerical Mathematics and Advanced Applications, pages 645-652. Springer, 2013.

[13] D. Göddeke and R. Strzodka. Cyclic reduction tridiagonal solvers on GPUs applied to mixed-precision multigrid. IEEE T. Parallel. Distr. 22(1):22-32, 2011.

[14] V. Grandgirard, M. Brunetti, P. Bertrand, N. Besse, X. Garbet, P. Ghendrih, G. Manfredi, Y. Sarazin, O. Sauter, E. Sonnendrücker, J. Vaclavik, and L. Villard. A drift-kinetic Semi-Lagrangian 4D code for ion turbulence simulation. J. Comput. Phys., 217(2):395-423, 2006.

[15] A.J. Klimas and W.M. Farrell. A splitting algorithm for Vlasov simulation with filamentation filtration. J. Comput. Phys., 110(1):150163,1994

[16] M.O. Lam, J.K. Hollingsworth, B.R. de Supinski, and M.P. LeGendre. Automatically adapting programs for mixed-precision floating-point computation. In Proceedings of the 27th international ACM conference on on supercomputing, pages 369-378. ACM, 2013.

[17] S. Le Grand, A.W. Götz, and R.C. Walker. SPFP: Speed without compromise - A mixed precision model for GPU accelerated molecular dynamics simulations. Comput. Phys. Commun., 184(2):374-380, 2013.

[18] W. Lei, Z. Yunquan, Z. Xianyi, and L. Fangfang. Accelerating Linpack performance with mixed precision algorithm on CPU+GPGPU heterogeneous cluster. In Computer and Information Technology (CIT), 2010 IEEE 10th International Conference on, pages 1169-1174. IEEE, 2010. 
[19] J.M. Qiu and C.W. Shu. Positivity preserving semi-Lagrangian discontinuous Galerkin formulation: theoretical analysis and application to the Vlasov-Poisson system. J. Comput. Phys., 230(23):8386-8409, 2011.

[20] J.A. Rossmanith and D.C. Seal. A positivity-preserving high-order semi-Lagrangian discontinuous Galerkin scheme for the Vlasov-Poisson equations. J. Comput. Phys., 230(16):6203-6232, 2011.

[21] F. Rozar, G. Latu, and J. Roman. Achieving memory scalability in the GYSELA code to fit exascale constraints. In Parallel processing and applied mathematics, pages 185-195. Springer, 2013.

[22] E. Sonnendrücker, J. Roche, P. Bertrand, and A. Ghizzo. The semiLagrangian method for the numerical resolution of the Vlasov equation. J. Comput. Phys., 149(2):201-220, 1999.

[23] C. Steiner, M. Mehrenberger, and D. Bouche. A semi-Lagrangian discontinuous Galerkin convergence. HAL preprint, hal-00852411, 2013. 